\title{
Modelling of Power Loss in Electrical Steels
}

\author{
I. Kucuk, K. Erturk, M.C. Hacissmailoglu \\ AND N. DEREBASI \\ Physics Department, Faculty of Arts and Sciences, Uludag University \\ Gorukle Campus, 16059 Bursa, Turkey \\ This paper presents a new artificial neural network approach based on \\ loss separation model to compute power loss on different types of electrical \\ steels. The network was trained by a Levenberg-Marquardt algorithm. The \\ results obtained by using the proposed model were compared with a com- \\ monly used conventional model. The comparison has shown that the neural \\ network model is in good agreement with experimental data with respect to \\ the conventional model.
}

PACS numbers: 75.50.Bb, 84.35.+i

\section{Introduction}

Thousands of tons electrical steels are used annually in wound cores for ultimate use in electromagnetic devices. A rapid and accurate prediction of power loss in electrical steels is becoming more important in their design and specifications for many reasons [1]. An artificial neural network (ANN) approach was very popular in the last years to contribute learning and generalization ability, fast real-time operation, and ease of implementation [2]. Recently the dynamic hysteresis loop, the power loss and permeability have been estimated by the ANN using the trained experimental data. The conventional methods are widely used for loss prediction but acceptable results are not guaranteed. However, the computational methods may provide good solutions to problems, which cannot be solved by a conventional method.

Specific power losses in soft magnetic materials can be divided into three components; static hysteresis, classical eddy current and anomalous losses [3]. The total specific power losses $P$ can be expressed by

$$
P=P_{\mathrm{h}}+P_{\mathrm{e}}+P_{\mathrm{a}},
$$

where $P_{\mathrm{h}}, P_{\mathrm{e}}$, and $P_{\mathrm{a}}$ are hysteresis, eddy current, and anomalous losses, respectively. 
Hysteresis loss $P_{\mathrm{h}}(\mathrm{W} / \mathrm{kg})$ and the anomalous loss $P_{\mathrm{a}}(\mathrm{W} / \mathrm{kg})$ per cycle under sinusoidal excitation can be expressed by [3]:

$$
\begin{aligned}
& P_{\mathrm{h}} / f=C_{0} B_{\mathrm{m}}^{x}, \\
& P_{\mathrm{a}} / f=C_{1} B_{\mathrm{m}}^{3 / 2} f^{1 / 2},
\end{aligned}
$$

where $C_{0}$ and $C_{1}$ are the unknown coefficients of the hysteresis loss and anomalous losses, respectively and $B_{\mathrm{m}}(\mathrm{T}), f(\mathrm{~Hz})$ and $x$ are the peak flux density, magnetizing frequency, and the Steinmetz parameter, respectively. The eddy-current losses $P_{\mathrm{e}}(\mathrm{W} / \mathrm{kg})$ per cycle under sinusoidal excitation can be calculated by the well-known formula

$$
\frac{P_{\mathrm{e}}}{f}=\frac{\pi^{2} t^{2} B_{\mathrm{m}}^{2} f}{6 \rho d},
$$

where $t(\mathrm{~m}), \rho(\Omega \mathrm{m})$, and $d\left(\mathrm{~kg} / \mathrm{m}^{3}\right)$ are the thickness of the electrical steel strip, the resistivity, and density of the material, respectively.

In this work, the power losses are modelled by determination of unknown coefficients in Eqs. (1)-(4) using an ANN and a set of experimental data [4]. The proposed model is not time consuming and more accurately and easily estimated.

\section{Neural network}

A neural network is an interconnected assembly of simple processing elements, units or nodes, whose functionality is loosely based on the human neuron. The processing ability of the network is stored in the inter-unit connection strengths or weights, obtained by a process of adaptation to, or learning from, a set of training patterns. Implicit knowledge is built into a neural network by training it. Some neural networks can be trained by being presented with typical input patterns and the corresponding expected output patterns. The error between the actual and expected outputs is used to modify the strengths, or weights, of the connections between the neurons. The Levenberg-Marquardt algorithm is used in this study. The Levenberg-Marquardt algorithm is a least-squares estimation algorithm based on the maximum neighborhood idea. Let $E(w)$ be an objective error function made up of $m$ individual error terms $e_{i}^{2}(w)$ as follows:

$$
E(w)=\sum_{i=1}^{m} e_{i}^{2}(w)=\|g(w)\|^{2},
$$

where $e_{i}^{2}(w)=\left(y_{\mathrm{d} i}-y_{i}\right)^{2}, y_{\mathrm{d} i}$ is the desired value of output neuron $i, y_{i}$ is the actual output of that neuron and $g(w)$ is a function containing the individual weight vector $w$ such that $E(w)$ is minimum. Using the Levenberg-Marquardt algorithm, a new weight vector $w_{k+1}$ can be obtained from the previous weight vector $w_{k}$ as follows:

$$
w_{k+1}=w_{k}+\delta w_{k},
$$

where $\delta w_{k}$ is defined as 


$$
\delta w_{k}=-\left(J_{k}^{\mathrm{T}} g\left(w_{k}\right)\right)\left(J_{k}^{\mathrm{T}} J_{k}+\lambda I\right)^{-1} .
$$

In Eq. (7), $J_{k}$ is the Jacobian of $g\left(w_{k}\right)$ evaluated by taking derivative of $g\left(w_{k}\right)$ with respect to $w_{k}, \lambda$ is the Marquardt parameter, $I$ is the identity matrix [5].

\section{ANN applications to power losses of electrical steels}

The proposed technique involves training a neural network to calculate the unknown hysteresis and anomalous coefficients $C_{0}$ and $C_{1}$ of the electrical steels when the values of $\rho, t, f, B$, and $P$ are given. Training an ANN using the Levenberg-Marquardt algorithm to compute $C_{0}$ and $C_{1}$ involves presenting them with different sets $(\rho, t, f, B$, and $P)$ sequentially and/or randomly and corresponding calculated values $C_{0}$ and $C_{1}$. Differences between the target outputs $\left(C_{0}\right.$ and $\left.C_{1}\right)$ and the actual outputs $\left(C_{0_{\mathrm{ANN}}}\right.$ and $\left.C_{1_{\mathrm{ANN}}}\right)$ of the ANN are calculated through the network to adapt its weights using Eq. (5)-(7). The adaptation is carried out after the presentation of each set $(\rho, t, f, B$, and $P)$ until the calculation accuracy of the network is deemed satisfactory according to some criterion (for example, when the errors between $C_{0}$ and $C_{0_{\mathrm{ANN}}}$ and $C_{1}$ and $C_{1_{\mathrm{ANN}}}$ for all the training set fall below a given threshold) or the maximum allowable number of epochs is reached. In order to understand the networks prediction accuracy and generalization capacity the networks were trained with the training set, cross validation set and checked with test data. Cross validation is a highly recommended criterion for stopping the training of a network. When the error in the cross validation increases the training should be stopped. The networks trained by the Levenberg-Marquardt algorithm was made in 500 epochs since the increase in cross validation error becomes stable at 500 epochs. For the validation, untrained previous experimental data are also used to test the neural model as well. The average correlation and prediction error were found to be $99 \%$ and $1 \%$ for the tested electrical steels, respectively.

\section{Results and discussion}

Two different electrical steels, which are commercially named Unisil (27M3) and Unisil (30M5), were used for the experimental validation of the proposed model. The estimates of $P$ were found to be in a range from $0.0003 \mathrm{~J} / \mathrm{kg}$ to $0.0014 \mathrm{~J} / \mathrm{kg}$ by use of estimated loss results in the electrical steels at $1 \mathrm{kHz}$. Figsures $1 \mathrm{a}$ and $\mathrm{b}$ show variations of predicted and measured specific losses with magnetic induction in the Unisil (27M3) and Unisil (30M5) electrical steels. Figures 1a and $\mathrm{b}$ show variations of predicted and measured specific losses with magnetic induction in the Unisil (27M3) and Unisil (30M5) electrical steels. In these strips specific total losses predicted by ANN are in good agreement with experimental results.

According to these results loss predictions by the ANN generate a better agreement with experimental data than the use of classical curve-fitting method, 

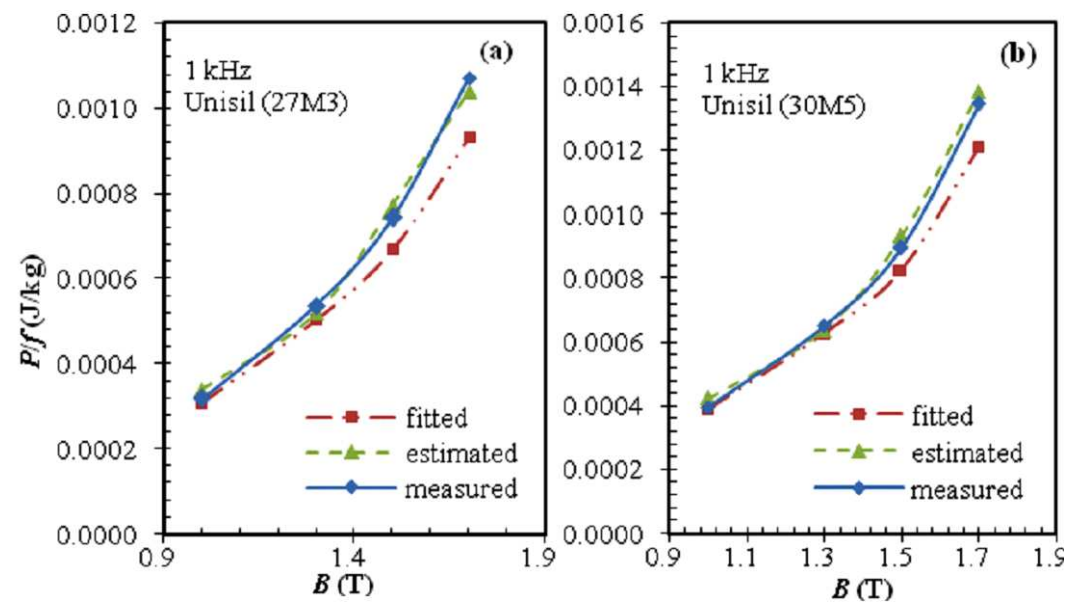

Fig. 1. Variation of estimated and measured losses in the (a) Unisil (27M3) and (b) Unisil (30M5).

particularly for high magnetic induction. Hence ANN should be used for loss predictions rather than the widely used curve-fitting method.

\section{Conclusions}

The predicted results from the ANN sound very satisfactory and in agreement with experimental results concerning the Unisil electrical steels. The model is fast and allows the application of standard learning algorithms for the neural network. Finally, this model capable of more accurately predicting power loss is also very useful to manufacturer working in this field. a

\section{References}

[1] I. Kucuk, N. Derebasi, Physica B, Condens. Matter 372, 260 (2006).

[2] S. Haykin, A Comprehensive Foundation, Macmillan, New York 1994.

[3] G. Bertotti, IEEE Trans. Magn. 24, 621 (1988).

[4] N. Tutkun, J. Magn. Magn. Mater. 300, 506 (2006).

[5] F. Wang, V.K. Devabhaktuni, C. Xi, Q. Zhang, Int. J. RF Microwave ComputerAided Eng. 9, 216 (1999). 